\title{
A Note on the Numerical Representation of Surface Dynamics in Quasigeostrophic Turbulence: Application to the Nonlinear Eady Model
}

\author{
Ross Tulloch AND K. SHAFER SMith \\ Center for Atmosphere Ocean Science, Courant Institute of Mathematical Sciences, New York University, New York, New York
}

(Manuscript received 8 August 2008, in final form 14 October 2008)

\begin{abstract}
The quasigeostrophic equations consist of the advection of linearized potential vorticity coupled with advection of temperature at the bounding upper and lower surfaces. Numerical models of quasigeostrophic flow often employ greater (scaled) resolution in the horizontal than in the vertical (the two-layer model is an extreme example). In the interior, this has the effect of suppressing interactions between layers at horizontal scales that are small compared to $N \delta z / f$ (where $\delta z$ is the vertical resolution, $N$ the buoyancy frequency, and $f$ the Coriolis parameter). The nature of the turbulent cascade in the interior is, however, not fundamentally altered because the downscale cascade of potential enstrophy in quasigeostrophic turbulence and the downscale cascade of enstrophy in two-dimensional turbulence (occurring layerwise) both yield energy spectra with slopes of -3 . It is shown here that a similar restriction on the vertical resolution applies to the representation of horizontal motions at the surfaces, but the penalty for underresolving in the vertical is complete suppression of the surface temperature cascade at small scales and a corresponding artificial steepening of the surface energy spectrum. This effect is demonstrated in the nonlinear Eady model, using a finite-difference representation in comparison with a model that explicitly advects temperature at the upper and lower surfaces. Theoretical predictions for the spectrum of turbulence in the nonlinear Eady model are reviewed and compared to the simulated flows, showing that the latter model yields an accurate representation of the cascade dynamics. To accurately represent dynamics at horizontal wavenumber $K$ in the vertically finite-differenced model, it is found that the vertical grid spacing must satisfy $\delta z \lesssim 0.3 f /(N K)$; at wavenumbers $K>0.3 f /(N \delta z)$, the spectrum of temperature variance rolls off rapidly.
\end{abstract}

\section{Introduction}

Synoptic-scale eddying flows in both the atmosphere and ocean lie in an asymptotic range well described by the quasigeostrophic $(\mathrm{QG})$ equations. Two phenomenological theories are typically invoked to describe the turbulent dynamics of such flows: geostrophic turbulence (GT; Charney 1971) and surface quasigeostrophic turbulence (SQG; Blumen 1978a; Held et al. 1995). These two theoretical ideas have been taken to describe complementary but distinct flows, with the former assumed to be more generic and relevant to atmospheric and oceanic motions.

Geostrophic turbulence is based on the analogous structure of QG and two-dimensional dynamics and leads to the prediction that total energy cascades to

Corresponding author address: K. Shafer Smith, Courant Institute of Mathematical Sciences, New York University, 251 Mercer St., New York, NY 10012.

E-mail: shafer@cims.nyu.edu large scales, both horizontally and vertically, whereas potential enstrophy cascades to small scales. Barotropization and the upscale cascade are widely observed phenomena, and their existence has put GT on firm ground. Geostrophic turbulence, however, neglects boundaries entirely in the downscale cascade-this is explicitly stated in Charney's list of six limitations of the theory proposed in his 1971 paper. Surface quasigeostrophy, by contrast, considers only boundary dynamics: it is derived by assuming a semi-infinite volume with constant potential vorticity (PV), bounded above or below by a surface on which temperature anomalies are advected by a geostrophic velocity field. The relationship between streamfunction and temperature is obtained by solving the homogeneous elliptic problem for the streamfunction in the interior, subject to a boundary condition given by the temperature field on the surface. Moreover, the conserved invariants, turbulent cascades, and spectral slopes are different in SQG and GT. In geostrophic turbulence, 
total energy cascades upscale and potential enstrophy downscale, yielding an energy spectrum with a $-5 / 3$ slope above the forcing range and a -3 slope in the downscale cascade range. In SQG the invariants are the total energy and temperature variance: total energy still undergoes an upscale cascade, but with a spectral slope of -2 , and the temperature variance [proportional to kinetic energy (KE) at the surface] cascades to small scales with a $-5 / 3$ spectral slope (Pierrehumbert et al. 1994).

With two boundaries bounding a region of constant $\mathrm{PV}$, one obtains the system on which the linear baroclinic instability problem of Eady (1949) is based. The fully nonlinear system of this type was considered by Blumen (1978a). The two-level Phillips model (Phillips 1954) is simultaneously taken as a representation of the Eady model [because their baroclinic instability growth rates are very similar; see, e.g., Pedlosky (1987)] and used as a model for geostrophic turbulence [because its downscale cascade yields a kinetic energy spectrum slope that is close to -3; see, e.g., Larichev and Held (1995)]. However, as argued by Blumen (1978a) and demonstrated numerically by Hoyer and Sadourny (1982), the turbulent dynamics that arise in the Eady and Phillips models are not equivalent.

It is straightforward to see that the Eady model will behave differently than the Phillips model (or Charney's geostrophic turbulence predictions) at small scales. In the Phillips model, for motion at horizontal wavenumber $K$ such that $f(K N)$ is less than the separation $H / 2$ between the two levels, the vortex stretching term is suppressed and the system becomes a set of decoupled layers, each controlled by two-dimensional vorticity dynamics. However, because both geostrophic turbulence and two-dimensional turbulence predict energy spectra with slopes of -3 , the vertical decoupling does not affect the energy density at a given horizontal scale; it only inhibits transfers of energy in the vertical. The same result holds in the two-mode (barotropic-baroclinic) formulation (see, e.g., Salmon 1980): the baroclinic flow becomes dominated by vorticity dynamics when $K \gg$ $N H / f$. In the Eady model, by contrast, at wavenumber $K$ such that the corresponding vertical scale $f(K N) \ll H$, the surface temperature perturbations will not "feel" the other boundary, and so the system will behave as a set of uncoupled SQG models, with $-5 / 3$ energy spectral slopes at each surface. Thus, no break in the spectrum at the surfaces is expected.

The Phillips model is a vertically finite-differenced QG model with only two levels, yet when the vertical resolution is increased, keeping the stratification and shear constant, the finite-difference model approaches a representation of the Eady model. This representation, however, is only accurate up to a horizontal wavenumber that depends on the vertical resolution of the model, for the following reasons. Temperature $\theta$ in the quasigeostrophic approximation is related to the vertical derivative of the streamfunction as $g \theta / \theta_{0}=f \psi_{z} \simeq f \delta \psi / \delta z$, where $\delta \psi / \delta z$ is the finite-difference approximation to $\psi_{z}$ and $g$ is gravitational acceleration. Because in geostrophic flow vertical and horizontal scales are linked by the Prandtl ratio $N / f$, horizontal temperature signals will only be accurately represented at wavenumbers sufficiently smaller than $K_{\delta z}=f /(N \delta z)$. Surface dynamics, dominated by temperature advection, should therefore be absent from low-vertical resolution QG simulations and, in general, only partially represented up to wavenumbers of order $f$ l $(N \delta z)$. Simulations reported on in this note confirm this expectation and provide a quantitative bound on the resolution necessary to resolve surface temperature $d y$ namics at a given horizontal wavenumber.

The differences between the Eady and Phillips models are frequently neglected. This is possibly due to the fact that at large scales, the models behave similarly, and so their subsequent accidental conflation has led to a neglect of their differences at smaller scales. Low vertical resolution in general, and the two-layer model in particular, are well rationalized by the idea of barotropization, which stems from Charney's theory of geostrophic turbulence (Charney 1971): if large-scale eddies are the result of an upscale cascade in both horizontal and vertical (scaled by $N / f$ ) dimensions, only the gravest few vertical modes should be necessary to represent those eddies (of course, similar logic should lead one to neglect higher horizontal wavenumbers as well). Moreover, the two-layer model has proved to be a tremendously useful tool for understanding much about atmospheric and oceanic eddy energy cycles and equilibration. In fact, one could say that so much has been understood through the lens of the two-layer model that those mesoscale phenomena that cannot be explained by it are often assumed to lie outside the realm of the quasigeostrophic approximation. A good example is the atmospheric energy spectrum (Nastrom and Gage 1985), and in a companion paper to this one (Tulloch and Smith 2009) we show that quasigeostrophic dynamics are adequate to explain those observations when surface effects are accounted for theoretically and resolved numerically.

\section{Vertical finite-difference representations of the PV streamfunction inversion}

The quasigeostrophic equations driven by a mean zonal baroclinic wind, and bounded above and below by rigid surfaces consist of a conservation equation for the QG potential vorticity and two advection equations for 
the potential temperature at each surface [equations are stated in numerous other works; see, e.g., Vallis (2006) or Tulloch and Smith (2009)]. The potential vorticity $q$ and potential temperature $\theta$ (scaled by $g / \theta_{0}$ ) are related to the streamfunction $\psi$ by the elliptic problem

$$
q=\nabla^{2} \psi+\left(\frac{f^{2}}{N^{2}} \psi_{z}\right)_{z}, \quad \text { and }\left.\quad \theta\right|_{z=0, H}=\left.f \psi_{z}\right|_{z=0, H} .
$$

A typical direct vertical discretization of this relation (assuming constant $N^{2}$ and grid spacing $\delta z$ ) is

$\Gamma_{n m} \psi_{m}=\frac{f^{2}}{N^{2}} \frac{1}{\delta z^{2}}\left\{\begin{array}{cc}\psi_{2}-\psi_{1}, & n=1, \\ \psi_{n-1}-2 \psi_{n}+\psi_{n+1}, & n=2 \ldots \mathcal{N}-1, \\ \psi_{\mathcal{N}-1}-\psi_{\mathcal{N}}, & n=\mathcal{N},\end{array}\right.$

where $\mathcal{N}$ is the total number of layers (which should not be confused with $N$, the buoyancy frequency). Bretherton (1966) showed that instead of considering nonconstant $\psi_{z}$ at the upper and lower boundaries, one can equivalently consider constant potential temperature at the boundaries with $\delta$ sheets of potential vorticity just inside the boundaries, given by

$$
q(z)=-\left.\frac{f^{2}}{N^{2}} \psi_{z}\right|_{z=H^{-}} \delta(z-H)+\left.\frac{f^{2}}{N^{2}} \psi_{z}\right|_{z=0^{+}} \delta(z) .
$$

The discrete operator $\Gamma_{n m}$ effectively includes an approximation of the $\delta$ sheets that is accurate to $O(N \delta z / f)$ - see appendix of Smith (2007)—so surface temperature dynamics at horizontal scales smaller than $O(N \delta z / f)$ are not captured.

\section{The structure of nonlinear Eady turbulence}

Here we consider the nonlinear Eady problem (Eady 1949): $f$ plane, uniform stratification and shear, with the fluid bounded above and below by rigid surfaces, depth $H$ apart. The mean interior PV gradient $Q_{y}=0$ and the mean surface temperature gradients are equal $-\Theta_{y}(H)=$ $\Theta_{y}(0)$-thus, $q=0$ and the motion is determined solely by temperature advection on the boundaries. Assuming horizontal periodicity and taking the Fourier transform of the remaining equations, one has

$$
\hat{\theta}_{t}+\hat{J}(\hat{\psi}, \hat{\theta})+i k\left(U \hat{\theta}+\hat{\psi}_{y}\right)=0, \quad z=0, H,
$$

with hatted variables denoting spectral components [e.g., $\psi(x, y, z, t)=\sum_{\mathbf{K}} \exp (i \mathbf{K} \cdot \mathbf{x}) \hat{\psi}_{\mathbf{K}}(z, t)$, with $\mathbf{K}=(k, l)$ the horizontal wavenumber vector], the hatted Jacobian denoting a double sum over wavenumbers of the spectral products, and the temperature-streamfunction relation being given by

$$
\begin{aligned}
\hat{\psi}(z, t)= & \frac{H}{\mu \sinh \mu}\left[\cosh \left(\mu \frac{z}{H}\right) \hat{\theta}(H, t)\right. \\
& \left.-\cosh \left(\mu \frac{z-H}{H}\right) \hat{\theta}(0, t)\right] .
\end{aligned}
$$

The variable $\mu=K N H / f$ is the nondimensional wavenumber and $K=|\mathbf{K}|$. We refer to this representation of Eady dynamics as the Blumen model, following Blumen (1978b).

One can understand the turbulent dynamics of the Eady model by considering the advection equations at each surface in the limits of large and small scales, separately. At the upper boundary $(z=H)$, the streamfunction is related to the surface temperatures as

$$
\hat{\psi}(H, t)=\frac{H}{\mu}\left[\frac{\hat{\theta}(H, t)}{\tanh \mu}-\frac{\hat{\theta}(0, t)}{\sinh \mu}\right] .
$$

At large scales $(\mu \ll 1)$, both sinh $\mu$ and tanh $\mu$ are approximated by $\mu$, so that $\hat{\psi}(H, t) \simeq\left(H / \mu^{2}\right)[\hat{\theta}(H, t)-$ $\hat{\theta}(0, t)]=-\left(H / \mu^{2}\right) \Delta \hat{\theta}$, where $\Delta \hat{\theta} \equiv \hat{\theta}(0, t)-\hat{\theta}(H, t)$. A similar relation exists at the bottom boundary, and therefore $\hat{\psi}(0, t) \simeq \hat{\psi}(H, t)$ at large scales. Furthermore, then, the temperature difference is related to the depthindependent streamfunction as $\Delta \hat{\theta}=-\mu^{2} \hat{\psi} / H$, and by subtracting the upper and lower advection equations, one has

$$
(\Delta \hat{\theta})_{t}+\hat{J}(\hat{\psi}, \Delta \hat{\theta})+i k\left(U \Delta \hat{\theta}+\hat{\psi} \Theta_{y}\right) \simeq 0 .
$$

Thus, the evolution of the temperature difference between the two surfaces is isomorphic to $2 \mathrm{D}$ vorticity flow at large scales.

At small scales $(\mu \gg 1)$, on the other hand, $\sinh \mu \rightarrow \infty$ but tanh $\mu \sim 1$, so that $\hat{\psi}(H, t) \simeq(H / \mu) \hat{\theta}(H, t)$, and similarly at the bottom. Thus, at small scales each surface obeys SQG dynamics, independent of the other surface.

In between these scale limits, where $\mu \sim 1$, baroclinic instability pumps energy into the eddying flow. Thus, the small-scale limit is governed by the downscale cascade, whereas the large-scale limit is controlled by the upscale cascade. Surface potential and kinetic energy in the upscale cascade of baroclinic turbulence and in the SQG downscale cascade are all expected to obey a $-5 / 3$ slope; thus, in nonlinear Eady turbulence there should be no spectral break in the surface energy spectra, and a $-5 / 3$ surface spectrum should dominate all scales. On the other hand, there should be a horizontal scale dependence in the interior flow. At large scale, temperature signals reach through the full depth of the domain, yielding a quasi-barotropic flow, and so the interior spectrum 


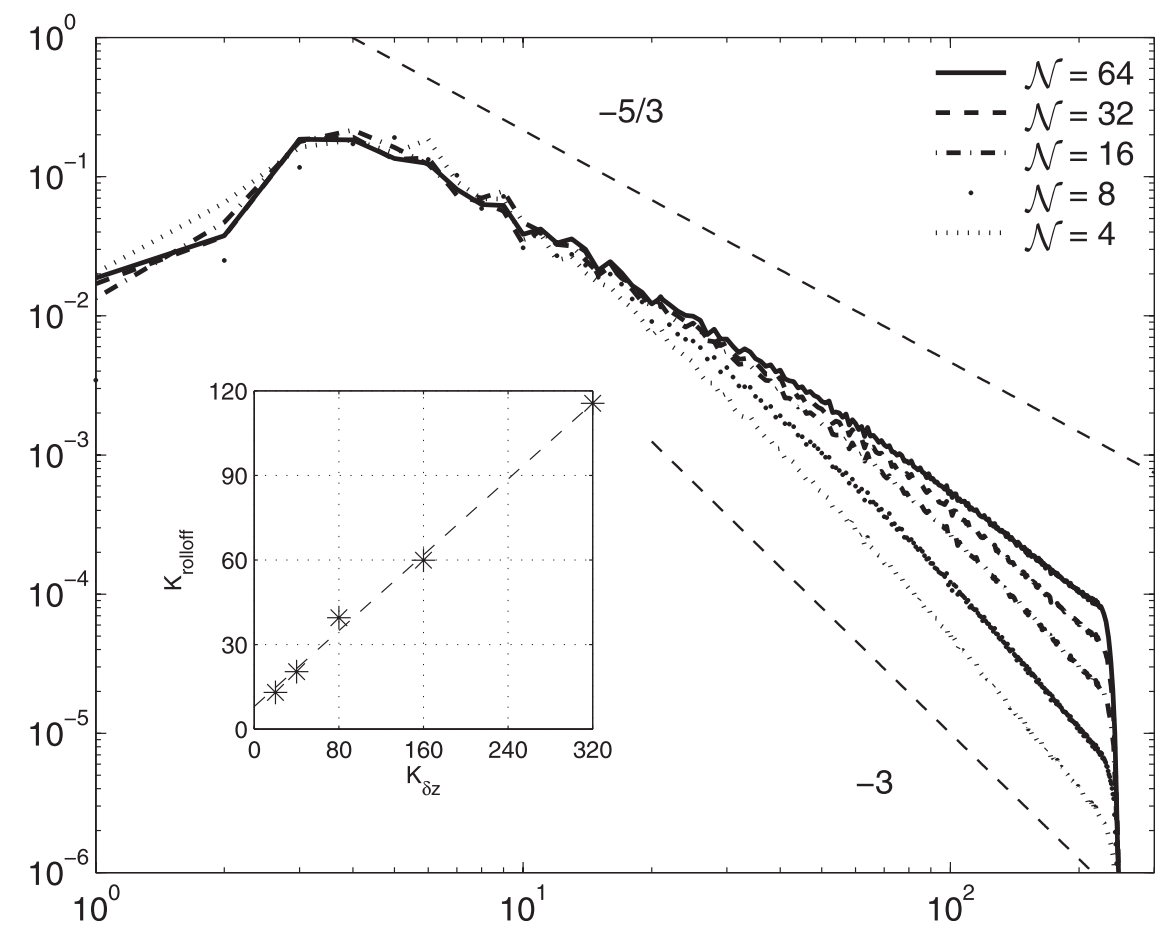

FIG. 1. Kinetic energy density vs horizontal wavenumber magnitude $K$ in the top layer of a series of Eady-forced QG simulations, with $\mathcal{N}=4,8,16,32,64$ layers, $\beta=0, U_{z}=1$, and deformation wavenumber $K_{d}=f L / N H=5$. Because there is no large-scale dissipation, the spectra shown are normalized by the baroclinic generation rate for each and then averaged in time between $t=4.5$ and $t=5$ for each simulation. The inset shows measured roll-off wavenumbers $K_{\text {roll-off }}\left(\right.$ where the spectral slope is $\left.K^{-7 / 3}\right)$ vs the prediction $K_{\delta z}=f /(N \delta z)$. The best-fit line (dashed line in figure) is $K_{\text {roll-off }}=0.34 K_{\delta z}+8$.

should also approach a $-5 / 3$ slope. At small scales, the temperature signals are trapped near their respective surfaces, and so the interior should be largely devoid of energy - the spectrum should roll off quickly as $\mu$ exceeds 1 and should be weakest in the middle of the domain.

\section{Numerical simulations}

We present a series of numerical simulations of quasigeostrophic turbulence with the Eady mean state, using two distinct representations of the vertical coupling: (i) advecting $q$ at every vertical level and using the finitedifference operator (2) to invert the streamfunction numerically and (ii) the "Blumen model," which advects only the upper and lower temperature fields and uses (4) to invert the streamfunction analytically. The calculations are performed in $\mathbf{K}$ space horizontally, with wavenumber 1 just filling the domain. The nonlinear terms are calculated using a dealiased fast Fourier transform. Downscale cascades are dissipated using a scale-selective exponential cutoff filter that acts explicitly on $K \geq 2 K_{\max } / 3$ but has little effect until the highest wavenumbers. The horizontal resolution of the simulations is $K_{\max }=511$, or $1024^{2}$ resolution in grid space. To preserve the dynamics and spectral slopes, the slow upscale cascades are not dissipated in this series of simulations.

Figure 1 shows wavenumber spectra of the kinetic energy density $K^{2}|\hat{\psi}|^{2}$ in the top level for simulations using the discretization in (2), with an increasing vertical resolution: $\mathcal{N}=4,8,16,32$, and 64 levels. All of the simulations have the same deformation scale $\left(K_{d} \equiv\right.$ $f L / N H=5)$ and seed energy $\left(E=10^{-3}\right)$ at $K_{0}=10$, which grows because of Eady baroclinic instability, leading to a dual cascade. The peak linear growth rate is near $1.6 K_{d} \approx 8$ and there is no linear baroclinic growth at wavenumbers above $2.4 K_{d} \approx 12$. Because there is no large-scale drag to halt the cascade and equilibrate the motion, we show a partial time average (from $t=4.5$ to $t=5$ in nondimensional time) of the KE density, normalized by $\epsilon^{2 / 3}$, where $\epsilon=U f^{2} / H N^{2}\left(\left.\overline{v \theta}\right|_{z=H}-\left.\overline{v \theta}\right|_{z=0}\right)$ is the baroclinic energy generation rate. The normalization nearly equalizes the peak amplitude of the spectra for each simulation, facilitating their comparison. Another consequence of the lack of drag is some variation of peak wavenumber among the spectra, but this is minor and irrelevant to the point addressed here. 


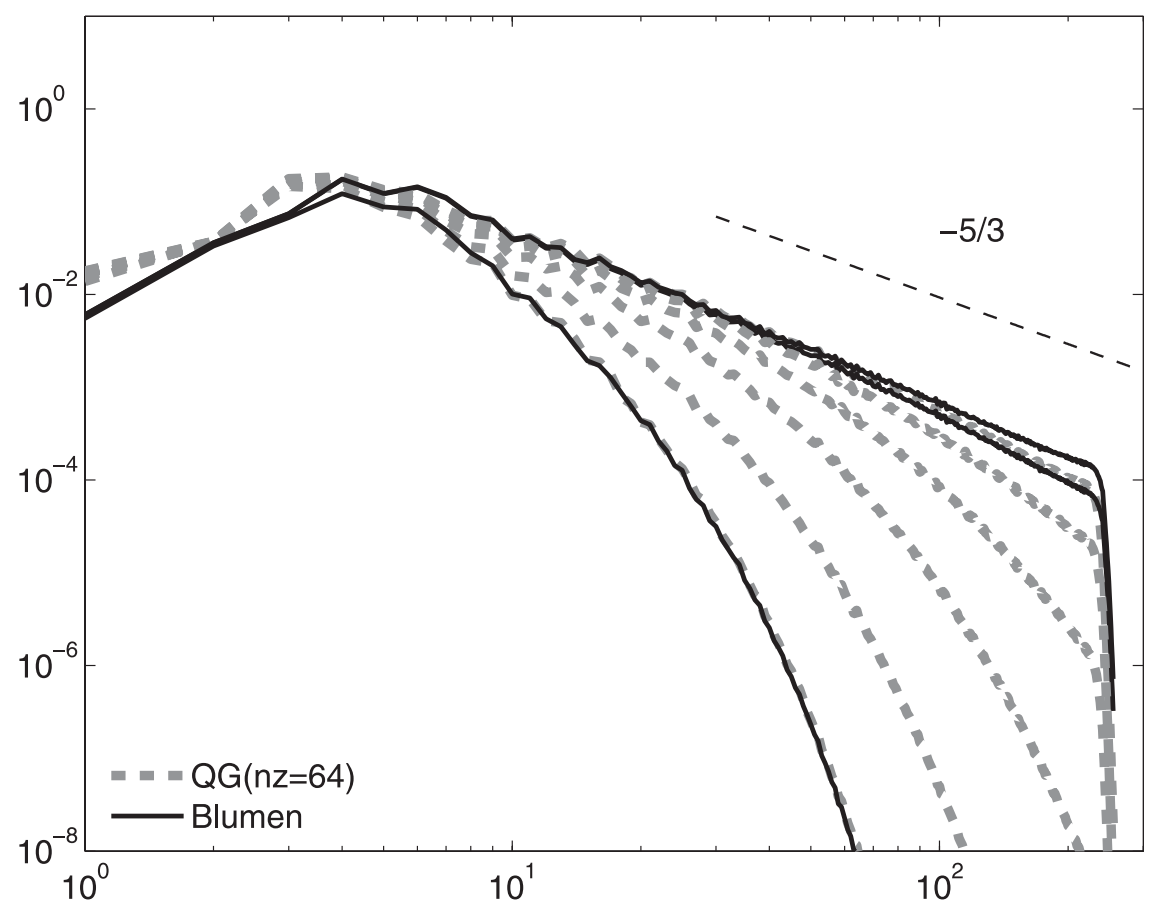

FIG. 2. Comparison of kinetic energy density in the Blumen model vs the standard QG formulation with 64 layers. The dashed gray lines are KE density at the mid-depths of layers $z_{1}, z_{2}, z_{4}, z_{8}, z_{16}$, and $z_{32}$ in the layered QG model, whereas the solid black lines are KE density at $z=0, z=z_{1}$, and $z=z_{32}$ in the Blumen model.

The kinetic energy spectra in the upper layer roll off from a $-5 / 3$ slope to a steeper slope, approaching -3 , but the wavenumber at which the roll-off begins increases with increasing vertical resolution. The inset of Fig. 1 plots the roll-off wavenumbers $K_{\text {roll-off }}$ (defined to be where the spectral slope of the KE spectrum drops to $\left.K^{-7 / 3}\right)$ against $K_{\delta z}=f /(N \delta z)$ for each $\delta z=H / \mathcal{N}$. The dependence of $K_{\text {roll-off }}$ on $K_{\delta z}$ is roughly linear, with a best-fit slope of 0.34 (given by the dashed line), indicating that to resolve a wavenumber $K$, a vertical grid scale spacing $\delta z \leq 0.34 f /(N K)$ is required.

The kinetic energy spectra at depths throughout the flow for both the 64-level simulation (dashed lines) and the Blumen model simulation (solid lines) are shown in Fig. 2. The Blumen simulation is normalized and averaged in the same way as the vertically discrete QG solution, and only three of its levels are plotted. The 64-level simulation is clearly a good representation of the nonlinear Eady model at this horizontal resolution; at higher horizontal resolutions, however, the discrete model will fail to resolve smaller horizontal scales at the surfaces unless its vertical resolution is concomitantly increased.

The spectrum of energy in the interior, as expected from (4), is determined solely by the horizontal cascade at the surfaces. Below the vertical trapping scale $f /(N K)$, there is essentially no energy in the flow. Because this vertical scale is dependent on horizontal wavenumber, the farther away from either surface one observes the spectrum, the larger the horizontal scale at which it begins to roll off. At midlevel, the spectrum roll-off begins practically at the deformation scale.

The implied resolution requirements suggested by the comparison of the Blumen and discrete representations of the nonlinear Eady model are similar to but different from those suggested by other authors. Barnier et al. (1991) argued, for example, that it is necessary to resolve horizontally the smallest baroclinic deformation scale. By contrast, the results here put the onus on the vertical resolution: whatever the horizontal resolution, the vertical resolution must be sufficiently fine for those horizontally included scales to accurately represent surface temperature dynamics. Lindzen and FoxRabinovitz (1989), on the other hand, argued that the vertical resolution must satisfy $\delta z \sim f \delta x / N$ to maintain stability in a numerical simulation of geostrophic flows, but we find no support for this claim (rather, here, as well as in the two-level model, failure to resolve vertical motions consistent with a given horizontal wavenumber merely removes some degrees of freedom). The closest analogy to the phenomenon discussed here is that studied by Solomon and Lindzen (2000), who demonstrated the necessity of sufficient resolution to model the 
barotropic instability of a point jet. Still, all such arguments imply the need for consistent horizontal and vertical resolutions, and all suggest their quotient should follow the Prandtl ratio, $N / f$.

\section{Discussion}

We have demonstrated that with sufficient vertical resolution, the numerical turbulent solution to the nonlinear Eady model with standard vertical finite-differencing converges to the "exact" solution, computed using a formulation proposed originally by Blumen (1978a) that explicitly advects the surface temperatures. This convergence results from the fact that in the standard vertical discretization, surface boundary conditions are effectively implemented as a finite approximation to the PV delta sheets of Bretherton (1966). The error of that approximation leads to a horizontal dependence on the vertical resolution: horizontal length scales on the order of the Prandtl ratio times the vertical grid spacing and smaller are not properly represented in the standard formulation.

In Tulloch and Smith (2009) we propose a generalized remedy to this problem that does not require excessive vertical resolution. The proposed formulation decomposes the QG streamfunction into a sum of interior and surface components that solve the associated elliptic inversion problems. Truncating the interior modes to a computationally manageable set yields an efficient and accurate representation of the full system at all depths. The formulation also allows for the generation of simplified models, including both well-known approximations and new ones.

In the ocean, recent simulations by Klein et al. (2008) indicate that the kinetic energy spectrum exhibits a shallowing near the surface, consistent with SQG effects near the surface. Scott and Wang (2005) observed the advective flux of surface kinetic energy to be in the upscale direction, and Capet et al. (2008) point out that the surface advective flux in SQG is also in the upscale direction. Although it does not seem likely that balanced surface dynamics can explain the entire picture, it is likely that it plays a part. In a forthcoming paper, we explore the degree to which a model that properly represents both the interior and surface dynamics can explain the ocean surface dynamics at the submesoscale.

Acknowledgments. We acknowledge helpful conversations with Glenn Flierl and Kevin Hamilton. This work was supported by NSF OCE-0620874.

\section{REFERENCES}

Barnier, B., C. L. Provost, and B. L. Hua, 1991: On the catalytic role of high baroclinic modes in eddy-driven large-scale circulations. J. Phys. Oceanogr., 21, 976-997.

Blumen, W., 1978a: Uniform potential vorticity flow: Part I. Theory of wave interactions and two-dimensional turbulence. J. Atmos. Sci., 35, 774-783.

_ 1978b: Uniform potential vorticity flow: Part II. A model of wave interactions. J. Atmos. Sci., 35, 784-789.

Bretherton, F. P., 1966: Critical layer instability in baroclinic flows. Quart. J. Roy. Meteor. Soc., 92, 325-334.

Capet, X., P. Klein, B. L. Hua, G. Lapeyre, and J. C. McWilliams, 2008: Surface kinetic energy transfer in SQG flows. J. Fluid Mech., 604, 165-174.

Charney, J. G., 1971: Geostrophic turbulence. J. Atmos. Sci., 28, $1087-1095$

Eady, E. T., 1949: Long waves and cyclone waves. Tellus, 1, 33-52.

Held, I. M., R. T. Pierrehumbert, S. T. Garner, and K. L. Swanson, 1995: Surface quasi-geostrophic dynamics. J. Fluid Mech., 282, $1-20$.

Hoyer, J.-M., and R. Sadourny, 1982: Closure modeling of fully developed baroclinic instability. J. Atmos. Sci., 39, 707-721.

Klein, P., B. L. Hua, G. Lapeyre, X. Capet, S. L. Gentil, and H. Sasaki, 2008: Upper ocean turbulence from high-resolution 3D resolution simulations. J. Phys. Oceanogr., 38, 17481763.

Larichev, V. D., and I. M. Held, 1995: Eddy amplitudes and fluxes in a homogeneous model of fully developed baroclinic instability. J. Phys. Oceanogr., 25, 2285-2297.

Lindzen, R. S., and M. Fox-Rabinovitz, 1989: Consistent horizontal and vertical resolution. Mon. Wea. Rev., 117, 25752583.

Nastrom, G. D., and K. S. Gage, 1985: A climatology of atmospheric wavenumber spectra of wind and temperature observed by commercial aircraft. J. Atmos. Sci., 42, 950-960.

Pedlosky, J., 1987: Geophysical Fluid Dynamics. 2nd ed. Springer, 710 pp.

Phillips, N. A., 1954: Energy transformations and meridional circulations associated with simple baroclinic waves in a twolevel, quasi-geostrophic model. Tellus, 6, 273-286.

Pierrehumbert, R. T., I. M. Held, and K. L. Swanson, 1994: Spectra of local and nonlocal two-dimensional turbulence. Chaos Solitons Fractals, 4, 1111-1116.

Salmon, R., 1980: Baroclinic instability and geostrophic turbulence. Geophys. Astrophys. Fluid Dyn., 15, 167-211.

Scott, R. B., and F. Wang, 2005: Direct evidence of an oceanic inverse kinetic energy cascade from satellite altimetry. J. Phys. Oceanogr., 35, 1650-1666.

Smith, K. S., 2007: The geography of linear baroclinic instability in Earth's oceans. J. Mar. Res., 65, 655-683.

Solomon, A., and R. S. Lindzen, 2000: The impact of resolution on a numerical simulation of barotropic instability. J. Atmos. Sci., 57, 3799-3816.

Tulloch, R. T., and K. S. Smith, 2009: Quasigeostrophic turbulence with explicit surface dynamics: Application to the atmospheric energy spectrum. J. Atmos. Sci., 66, 450-467.

Vallis, G. K., 2006: Atmospheric and Oceanic Fluid Dynamics: Fundamentals and Large-Scale Circulation. Cambridge University Press, 745 pp. 